\title{
Prognostic values of a novel multi-mRNA signature for predicting relapse of cholangiocarcinoma
}

\author{
Han Guo ${ }^{1 *}$, Jie Cai ${ }^{1 *}$, Xuan Wang ${ }^{2 *}$, Bingrui Wang ${ }^{1}$, Fang Wang ${ }^{2}$, Xiang $\mathrm{Li}^{1}$, Xiaoye $\mathrm{Qu}^{1}$, Xianming Kong ${ }^{4}$, \\ Yueqiu $\mathrm{Gao}^{2}$, Hailong $\mathrm{Wu}^{3}$, Xuehua Sun ${ }^{\circledR}{ }^{\bowtie}$, Qiang $\mathrm{Xia}^{1 凶}$, Xiaoni Kong ${ }^{1,2}$ \\ 1. Department of Liver Surgery, Renji Hospital, School of Medicine, Shanghai Jiao Tong University, Shanghai, China. \\ 2. Institute of Clinical Immunology, Department of Liver Diseases, Central Laboratory, ShuGuang Hospital Affiliated to Shanghai University of Chinese \\ Traditional Medicine, Shanghai, China \\ 3. Shanghai Key Laboratory for Molecular Imaging, Collaborative Research Center, Shanghai University of Medicine and Health Sciences, Shanghai, China. \\ 4. Central Laboratory, Renji Hospital, School of Medicine, Shanghai Jiao Tong University, Shanghai, China. \\ * These authors contribute equally to this work. \\ $\triangle$ Corresponding authors: Xiaoni Kong, PhD, Institute of Clinical Immunology, Department of Liver Diseases, ShuGuang Hospital Affiliated to Shanghai \\ University of Chinese Traditional Medicine, 528 Zhangheng Road, Shanghai, China, 201203. Telephone: +86 21 20256188. E-mail: xiaoni-kong@126.com; Or \\ Qiang Xia, MD, PhD, E-mail: xiaqiang@shsmu.edu.cn; Or Xuehua Sun, MD, E-mail: susan_sxh@sina.com.
}

(c) The author(s). This is an open access article distributed under the terms of the Creative Commons Attribution License (https://creativecommons.org/licenses/by/4.0/). See http://ivyspring.com/terms for full terms and conditions.

Received: 2019.07.29; Accepted: 2019.11.05; Published: 2020.01.16

\begin{abstract}
Cholangiocarcinoma (CCA) is an epithelial cancer and has high death and recurrence rates, current methods cannot satisfy the need for predicting cancer relapse effectively. So, we aimed at conducting a multi-mRNA signature to improve the relapse prediction of CCA. We analyzed mRNA expression profiling in large CCA cohorts from the Gene Expression Omnibus (GEO) database (GSE76297, GSE32879, GSE26566, GSE31370, and GSE45001) and The Cancer Genome Atlas (TCGA) database. The Least absolute shrinkage and selection operator (LASSO) regression model was used to establish a 7-mRNA-based signature that was significantly related to the recurrence-free survival (RFS) in two test series. Based on the 7-mRNA signature, the cohort TCGA patients could be divided into high-risk or low-risk subgroups with significantly different RFS [ $<<0.001$, hazard ratio (HR): 48.886, 95\% confidence interval $(\mathrm{Cl}):$ 6.226-3.837E+02]. Simultaneously, the prognostic value of the 7-mRNA signature was confirmed in clinical samples of Ren Ji hospital ( $\mathrm{p}<0.001$, HR: 4.558, 95\% Cl: 1.829-11.357). Further analysis including multivariable and sub-group analyses revealed that the 7-mRNA signature was an independent prognostic value for recurrence of patients with CCA. In conclusion, our results might provide an efficient tool for relapse prediction and were beneficial to individualized management for CCA patients.
\end{abstract}

Key words: cholangiocarcinoma; Gene Expression Omnibus database; least absolute shrinkage and selection operator model; mRNA signature; recurrence-free survival.

\section{Introduction}

Cholangiocarcinoma (CCA) is the second most common primary liver cancer worldwide [1-3]. During the past few decades, incidence and high recurrence rates for all CCA were closely correlated to poor outcomes [4-6]. Unfortunately, there is still no better way to accurately predict recurrence. Although TNM staging systems according to different versions of the American Joint Committee on Cancer (AJCC) had shown valuable but still insufficient for predicting relapse in different subtypes of CCA [5].
An increasing amount of evidence has demonstrated that messenger RNA (mRNA) as molecular biomarkers could promote the prognostic evaluation and identification of potential high-risk CCA patients $[7,8]$. For example, KRAS mutations were associated with deregulation of epidermal growth factor receptor (EGFR) and ERBB2 signaling network, derangement of genes participating in proteasomal activity could lead to poor prognosis [9]. However, many genes and signal pathways were also 
present in hepatocellular carcinoma, and single gene as a prognostic indicator for CCA was not rigorous enough. So, our study aimed at finding a multi-mRNA model to help better predicting the relapse of CCA patients.

GEO [10] and The Cancer Genome Atlas [11] are two main public databases that provided numerous array-based and sequence-based data for researchers. By using bioinformatic methods [12], we could obtain large amounts of data quickly and conveniently. Therefore, we identified significant genes that expressed differentially between CCA samples and normal bile duct tissue or para cancerous samples in five datasets from GEO and TCGA database, respectively. Then, we utilized the least absolute shrinkage and selection operator (LASSO) regression model $[13,14]$ and built a 7 -mRNA-based signature for predicting relapse. Cox regression and the time-dependent ROC curve demonstrated that this 7-mRNA-based signature had an excellent prediction for RFS. In addition, gene ontology (GO) enrichment analysis and Kyoto Encyclopedia of Genes and Genomes (KEGG) pathway analysis were performed for discovering essential marker and pathways. All of these may provide an efficient method to judge recurrence rate and was beneficial to individualized management for CCA patients.

\section{Materials and methods}

\section{Preparation of CCA datasets}

The gene expression data of CCAs were downloaded from Gene Expression Omnibus (GEO) and The Cancer Genome Atlas (TCGA) database. There were five appropriate CCA datasets from the GEO database (GSE76297, GSE32879, GSE26566, GSE31370, and GSE45001) met the following criteria: a). a total of more than 10 samples, including both tumor and non-tumor samples; b) annotated genes accounting for more than $90 \%$ of the total transcriptomes ( $\mathrm{n}>17000)$; and $\mathrm{c}$ ) the number of differentially expressed genes (DEGs) more than 100. Details of these five datasets were listed in Table 1. In addition, the TCGA database provided gene expression profiles from RNA-seq and corresponding clinical information in 36 CCA patients with RFS status. The online analytical tool GEO2R [12] was used to screen out DEGs between CCA and non-tumor samples in the GEO database, and we obtained DEGs from the TCGA database by using $R$ package "edgeR". Here, genes with adjusted p-value $<0.01$ and fold change $(\mathrm{FC})>1.5$ or $<-1.5$ were considered as DEGs.

Three gene expression profiles were utilized to recognize DEGs between tumor tissues and normal intrahepatic bile duct tissues or non-tumor tissues $(\mathrm{T} / \mathrm{N})$, and the other three gene expression profiles were used to recognize DEGs between tumor tissues and para-cancerous tissues $(\mathrm{T} / \mathrm{P})$. Next, overlapping analysis of these DEGs was conducted by website imageGP, DEGs within 2 series or more were regarded as credible DEGs in each Venn diagram. Finally, 194 DEGs among TvsN, TvsP and TCGA were identified, which including 87 up-regulated genes and 107 down-regulated genes.

Table 1. GEO datasets enrolled in the study.

\begin{tabular}{|c|c|c|c|c|c|}
\hline \multirow[t]{2}{*}{ Database } & \multirow[t]{2}{*}{ Source } & \multicolumn{3}{|c|}{ Sample } & \multirow[t]{2}{*}{ Platform } \\
\hline & & $\bar{T}$ & $\mathbf{P}$ & $\mathbf{N}$ & \\
\hline GSE26566 & $\begin{array}{l}\text { https://www.ncbi.nlm.nih.gov/geo } \\
\text { /query/acc.cgi?acc=GSE26566 }\end{array}$ & $\begin{array}{l}10 \\
6\end{array}$ & 59 & 6 & Illumina v2.0 \\
\hline GSE32879 & $\begin{array}{l}\text { https://www.ncbi.nlm.nih.gov/geo } \\
\text { /query/acc.cgi?acc=GSE32879 }\end{array}$ & 16 & - & 7 & $\begin{array}{l}\text { Affymetrix } \\
1.0 \text { ST }\end{array}$ \\
\hline GSE76297 & $\begin{array}{l}\text { https://www.ncbi.nlm.nih.gov/geo } \\
\text { / query/acc.cgi?acc=GSE76297 }\end{array}$ & 91 & 92 & - & $\begin{array}{l}\text { Affymetrix } \\
\text { HTA-2_0 }\end{array}$ \\
\hline GSE45001 & $\begin{array}{l}\text { https://www.ncbi.nlm.nih.gov/geo } \\
\text { /query/acc.cgi?acc=GSE45001 }\end{array}$ & 10 & 10 & - & $\begin{array}{l}\text { Agilent-02800 } \\
4\end{array}$ \\
\hline GSE31370 & $\begin{array}{l}\text { https://www.ncbi.nlm.nih.gov/geo } \\
\text { /query/acc.cgi?acc=GSE31370 }\end{array}$ & 6 & - & 5 & Illumina V4.0 \\
\hline
\end{tabular}

T: tumor tissues; P: para-cancerous tissues; $\mathrm{N}$ : normal intrahepatic bile duct tissues or non-tumor tissues.

\section{GO enrichment analysis and KEGG pathway analysis}

GFO analysis is a common genes and gene products annotating method, including biological processes (BP), cellular component (CC), molecular function (MF). The Kyoto Encyclopedia of Genes and Genomes (KEGG) database is a knowledge base for systematic analysis, annotation, and visualization of gene functions. In our study, the $\mathrm{R}$ package "clusterprofiler" was used to provide functional classification, and KEGG pathway, of the 87 up-regulated and 107 down-regulated DEGs, respectively. We listed the top 10 of all terms in every category, $\mathrm{p}<0.05$ was set as the cutoff point.

\section{Establishment of the LASSO regression model}

For these 194 candidate mRNAs, the optimal cutoff value of each mRNA was generated based on receiver operating characteristic (ROC) curve, and the area under the curve (AUC), sensitivities and specificities of these mRNAs were also obtained. Next, there were 127 genes with AUC $\geq 0.55$ remained. According to the cutoff value, 36 patients of the TCGA database were classified into high- or low-expression status according to each mRNA. Based on the expression status data of these 127 DEGs, we constructed LASSO COX regression models with the $\mathrm{R}$ package "glmnet". The least absolute shrinkage and selection operator (LASSO) is a most 
famous method for analyzing survival data, and especially suitable for analyzing gene expression profile, which has higher dimensionality, smaller sample size and strongly relevant variables[15, 16]. The "glmnet" package returned a sequence of models for us, the value of the tuning parameter $\lambda$ was negatively associated with the complexity of the model and the value of deviance. When the value of the invisible $\lambda$ increased from left to right, the number of nonzero coefficients increased accordingly. Ten-time cross validations were used to determine the optimal values of $\lambda$ and a value $\lambda=0.20770$ with $\log$ $(\lambda)=-0.68256$ was chosen by 10 -fold cross-validation via minimum criteria; a vertical line was drawn at L1 norm $=2.388$, which corresponds to the optimal value $=0.20770$. However, the results of the $\lambda$ value might be slightly variable during different times of analysis. So, 10-fold cross-validation was running up to 100 times and the cross-validated errors were averaged.

\section{Validation in Clinical CCA specimens}

Between January 1, 2012, and December 30, 2017, the human CCA tissues were obtained from the Department of Liver Surgery, Ren Ji Hospital, Shanghai Jiaotong University. Protocol and free of written informed consent were approved by the ethical review committee of Renji Hospital, School of Medicine, Shanghai Jiaotong University.

We excluded patients for the following criteria: combination with other tumors, perioperative mortality, preoperative radiotherapy and chemotherapy, conservative treatment and incomplete data. Finally, we obtained 44 patients' tissues and all tissues were pathologically confirmed. The clinicopathological features of the Ren Ji cohort were listed in Table S1. Tumor staging was assessed according to the 8th edition staging classification system of AJCC [17].

Follow up information of these CCA patients has received check-ups every 2-3 months during the first 2 years and every 3-6 months until May 2018. The RFS was calculated from the date of tumor resection until the detection of tumor recurrence, death from a cause other than CCA, or the last follow-up visit.

\section{Quantitative real-time PCR (qRT-PCR)}

Total RNA was extracted and reversed using the RNeasy Mini Kit (Qiagen, Valencia, CA) and the Revert Aid First Strand cDNA Synthesis Kit (Thermo Scientific, Rockford, IL), respectively. The expression of CD36, GGCX, UBASH3B, DBN1, PTTG1, CCNA2, SPATS2, and 18S mRNA were determined by qRT-PCR using SYBR Green PCR Master Mix, and Ct value was enrolled for data analysis. Related primers sequences were listed in Table S2. All these experiments were conducted according to the manufacture instructions.

\section{Statistical analysis}

The statistical analysis was carried out using SPSS 17.0 and GraphPad Prism 6 software. The optimal cutoff of risk score was determined when the sensitivity and specificity in the ROC curve [18, 19] achieved optimum for predicting recurrence-free survival. With this risk score cutoff, the patients were divided into high- or low-risk groups. Recurrence-free survival analysis between high- and low-risk groups was assessed by the Kaplan-Meier analysis and compared using the log-rank test. Time-dependent ROC curves were employed to demonstrate the predictive accuracy of different variables. Univariable and multivariable Cox analyses were performed to investigate whether the gene signature was independent of other clinicopathological characteristics, and Pearson chi-squared test or Fisher's exact test was used to examine the association between the clinicopathological characteristics and 7-mRNA signature. A difference was defined as significant at $\mathrm{P}<0.05$.

\section{Results}

\section{Identification of differentially expressed genes in cholangiocarcinoma from public datasets.}

Detailed information of the five eligible CCA datasets meets our criteria in the GEO database (GSE76297, GSE32879, GSE26566, GSE31370, and GSE45001) were shown in Table 1. After analyzing these CCA datasets using GEO2R, 4005, 6554, 990, 3893, 879 and 399 DEGs were respectively recognized in GSE76297-T/P, GSE26566-T/P, GSE45001-T/P, GSE32879-T/N， GSE26566-T/N， GSE31370-T/N (Figure 1A-F). DEGs shared within 2 series or more were regarded as credible DEGs in each Venn diagram, and 2666 and 422 credible DEGs were recognized in $\mathrm{T} / \mathrm{P}$ and $\mathrm{T} / \mathrm{N}$ groups respectively (Figure 1G-H). Similarly, 2545 DEGs meet the criteria with p-value $<0.01$ and FC $>1.5$ or $<-1.5$ were gathered in TCGA, including 1132 up-regulated and 1413 down-regulated genes (Figure S1). The Overlapping analysis was further performed between GEO and TCGA database, and 194 DEGs were identified, which were believed to be commonly dysregulated in CCA (Figure 1I).

In addition, GO and KEGG pathway enrichment analyses were conducted for these overlapping up- or down-regulated genes. As shown in Figure 2A-B, up-regulated genes were most enriched in organelle fission and cell cycle pathways by GO-BP and KEGG analyses, respectively. Contrarily, the organic 
hydroxy compound metabolic process and bile secretion pathway were respectively greatly enriched in GO-BP and KEGG analyses by down-regulated genes (Figure 2C-D). Meanwhile, enrichment analyses for cellular component (CC) and molecular functions (MF) were also performed (Figure S2).

\section{Construction of a 7-mRNA signature from the TCGA cohort}

For these 194 candidate DEGs, the optimal cutoff point was determined when the sensitivity and specificity of the ROC curve achieved optimum. According to each mRNA cutoff value, 36 patients were classified into high or low expression status. Besides, AUC $\geq 0.55$ was a restrictive condition for filtering some mRNAs that hardly had a prognostic value. Ultimately, $127 \mathrm{mRNAs}$ with $\mathrm{AUC} \geq 0.55$ were utilized to construct the LASSO COX regression model.

The "glmnet" package $[13,20]$ returned a sequence of models for us (Figure S3A), and 10-fold cross-validations were performed to select the best one. As shown in Figure 3A, a value $\lambda=0.20770$ with $\log (\lambda)=-0.68256$ was chosen by 10 -fold cross-validation via minimum criteria. However, the results of the $\lambda$ value might be slightly variable during different times of analysis. So, 10 -fold cross-validation was running up to 100 times and the cross-validated errors were averaged. Finally, the $\lambda$ with minimum mean cross validation error was still returned about 0.20770. At this $\lambda$ value, $7 \mathrm{mRNAs}$ including CD36, GGCX, UBASH3B, DBN1, PTTG1, CCNA2 and SPATS2 with nonzero coefficients were selected
A

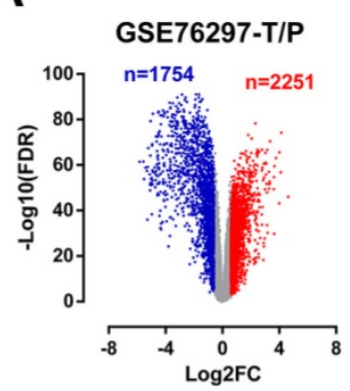

D

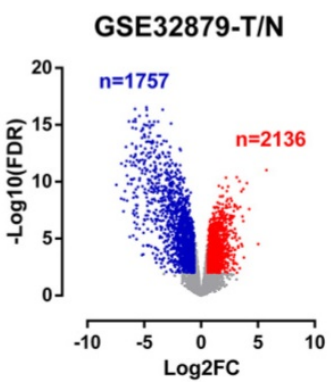

G

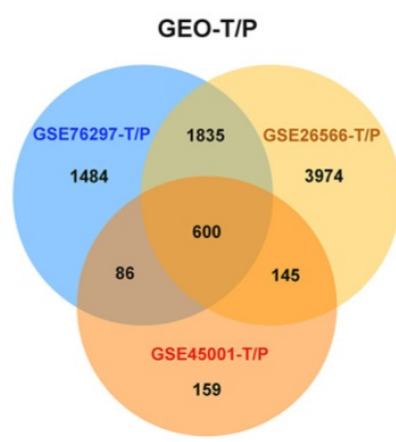

B

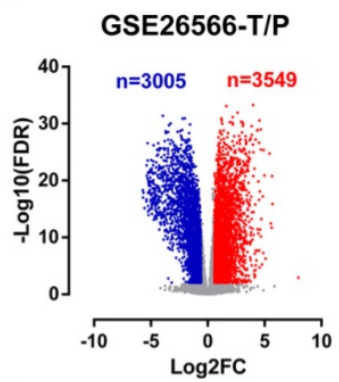

E

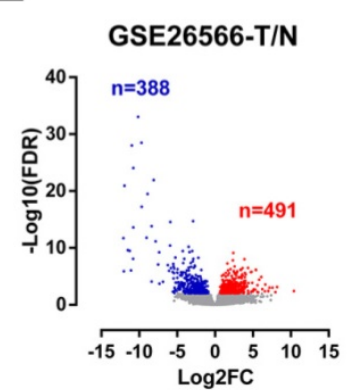

H

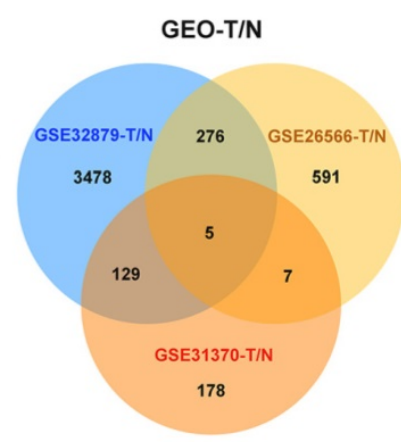

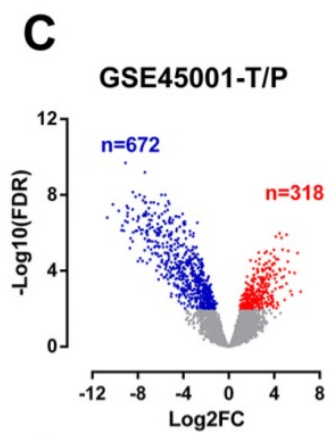

F

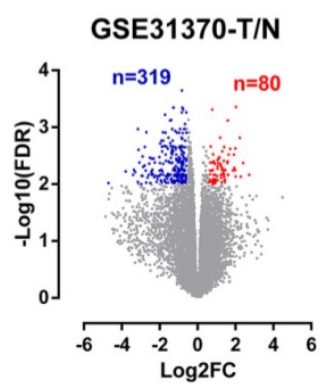

I

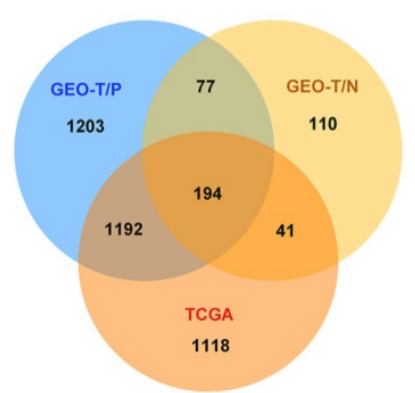

Figure 1. Identification of differentially expressed genes in cholangiocarcinoma from public CCA datasets. (A-F) Volcano plots of DEGs in the 5 indicated datasets. (X-axis: $\log 2$ (FC); Y-axis: -log10(FDR) for each gene. Genes with FDR $<0.01$ and FC $>1.5$ or $<-1.5$ were considered as DEGs in each series. Blue: down-regulated genes; Grey: non-differential genes; Red: up-regulated genes). (G-H) Overlapping analyses of DEGs in TvsP (G) and TvsN (H) groups, DEGs shared within 2 datasets or more were regarded as credible DEGs in each Venn diagram. (I) Overlapping analysis of GEO and TCGA datasets. 
(Figure 3B). Among them, CD36 and GGCX were down-regulated in CCA, and the other 5 genes were up-regulated. Meanwhile, person's correlation tests showed that the expression of these 7 genes was independent of each other (Figure S3B). Based on the expression status of these 7 mRNAs, a risk-score formula for RFS was constructed as follows: Risk score $=(-0.96873 \times$ expression status of CD36) +

\section{A}

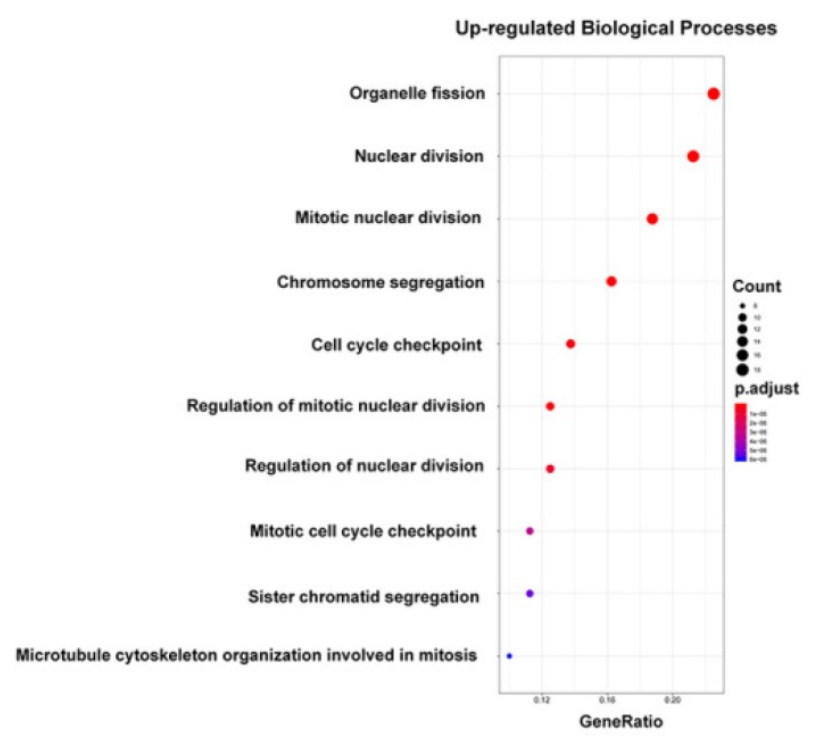

C

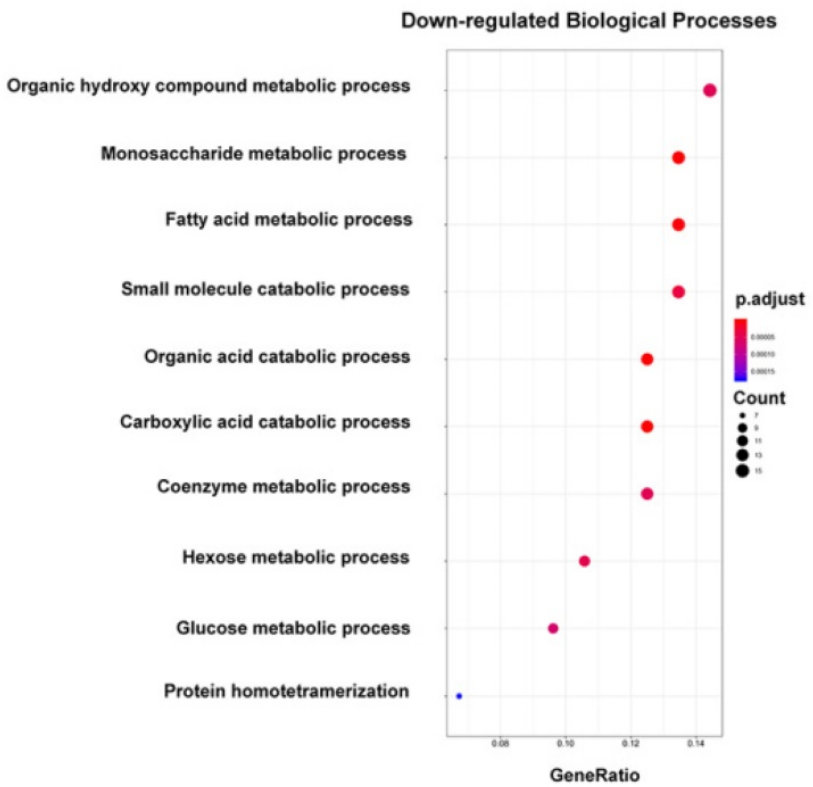

$(-0.03944 \times$ expression status of GGCX) $+(0.01064 \times$ expression status of UBASH3B $)+(0.04955 \times$ expression status of DBN1 $)+(0.24927 \times$ expression status of PTTG1 $)+(0.31598 \times$ expression status of CCNA2 $)+(0.57201 \times$ expression status of SPATS2 $)$. In the formula, low expression status was equivalent to 0 , and high expression status was equivalent to 1 .
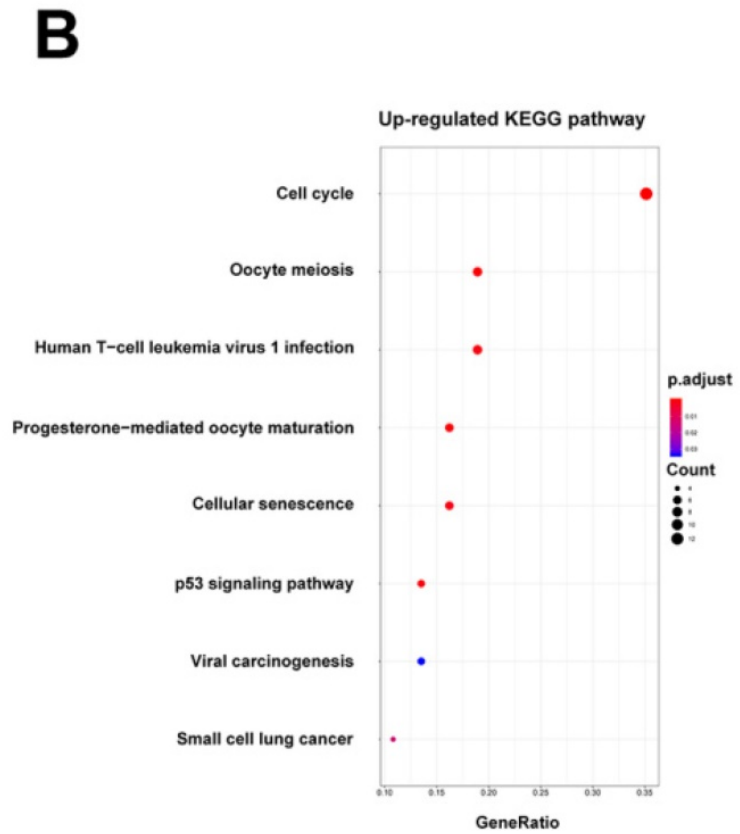

D

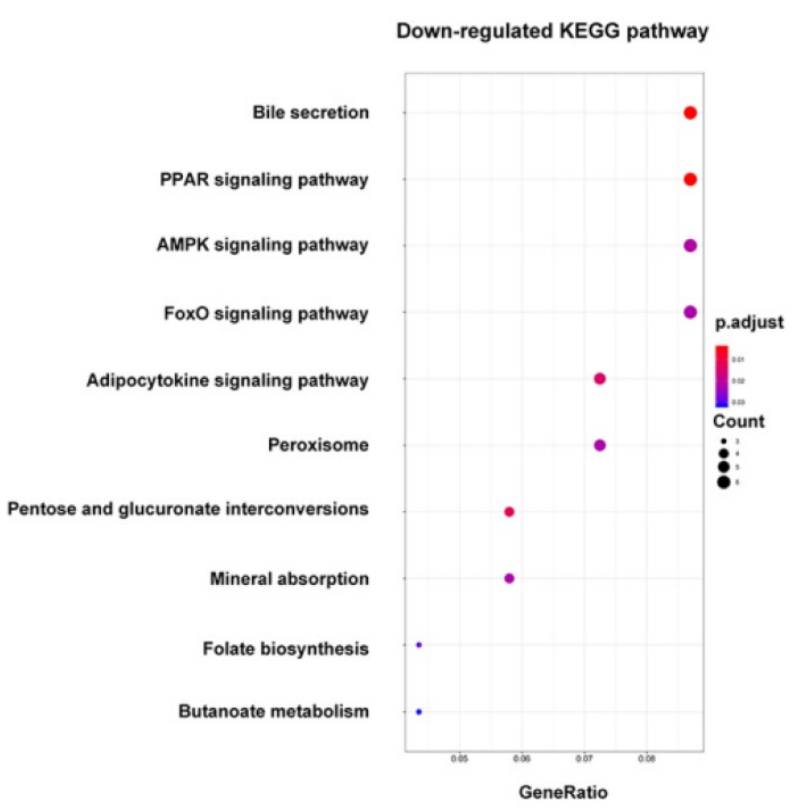

Figure 2. Biological processes (BP) enrichment analysis and KEGG pathway analysis. (A-B) GO biological processes (BP) enrichment analysis and KEGG pathway analysis of upregulated DEGs. (C-D) GO biological processes (BP) enrichment analysis and KEGG pathway analysis of downregulated DEGs. 
A

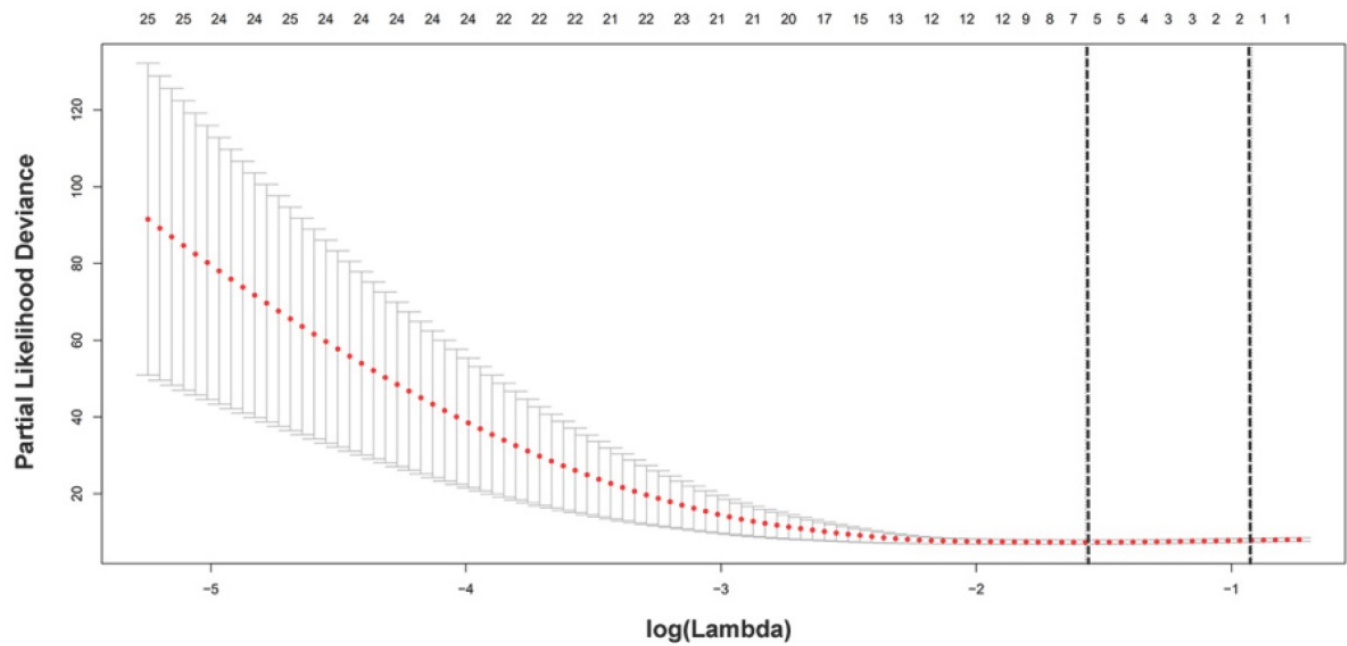

B

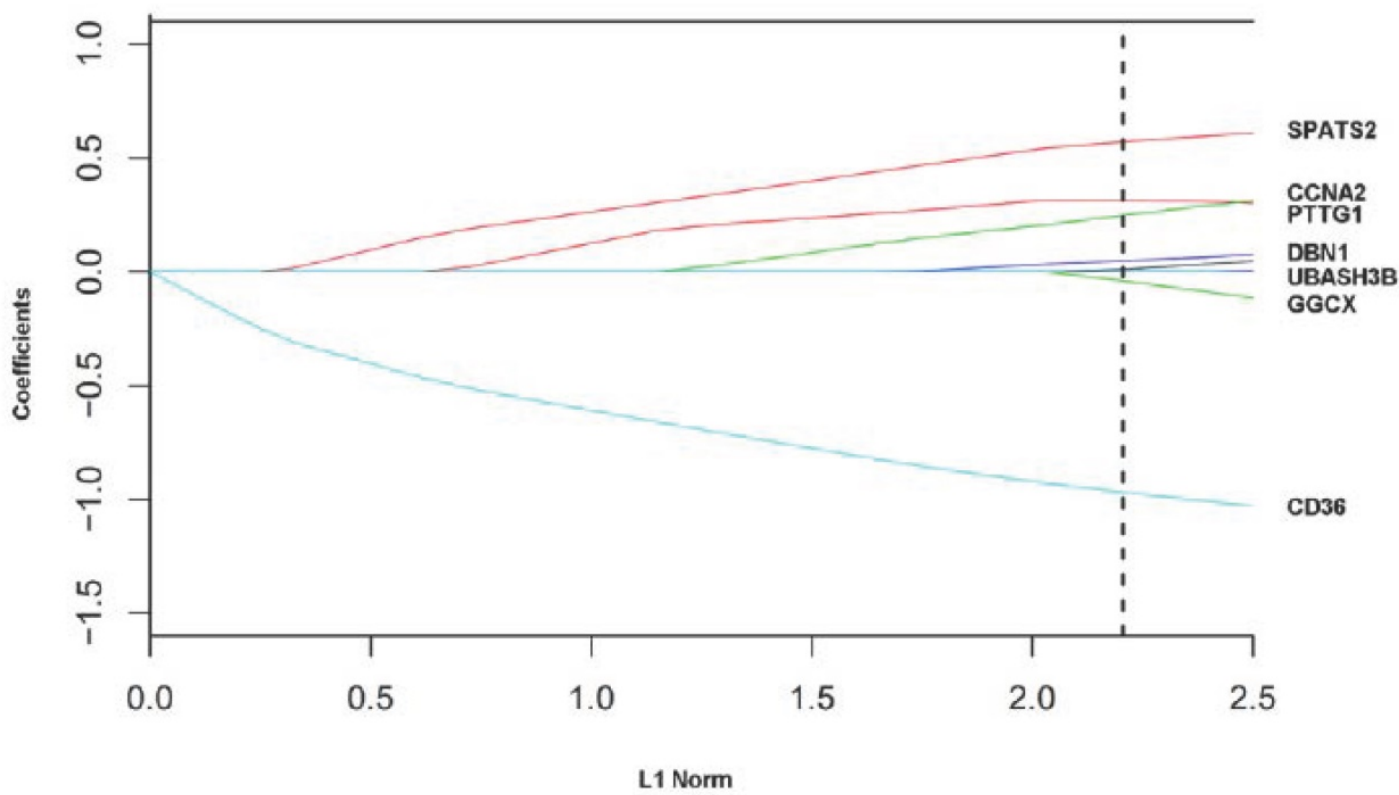

Figure 3. Construction of a 7-mRNA signature from the TCGA cohort. (A) 10-fold cross-validation for tuning parameter selection in the LASSO model. The dotted vertical lines are drawn at the optimal values by minimum criteria (lambda.min, left vertical dotted line) and 1 -SE criteria (lambda.1 se, right vertical dotted line). (B) LASSO model at optimal lambda value, 7 mRNAs with nonzero coefficients were selected.

\section{Evaluation of the risk score formula for relapse} in TCGA Cohort

Then, the risk scores for relapse were calculated for every patient in the TCGA Cohort. As shown in Figure $4 \mathrm{~A}$, the patients were more trended to relapse when the risk score increased. Patients were divided into high-risk $(n=17)$ or low-risk $(n=19)$ groups using the optimal risk score as the cutoff point. The Recurrence rate of the high-risk group was extremely increased throughout the study period until the analytical endpoint, at which $94.74 \%$ of patients in the high-risk group experienced CCA recurrence while only $0.58 \%$ of patients in low-risk group relapsed $(\mathrm{p}<$ 0.001 , Figure 4B). Kaplan-Meier analysis showed that CCA patients with higher risk score had significantly worse RFS than those with lower risk score (HR = 48.886, 95\% CI: 6.229-383.657, p < 0.001, Figure 4C). In addition, the time-dependent ROC curves between the 7-mRNA signature and RFS showed that AUC at 1 year, 3 years, 5 years, and $>5$ years were $0.973,0.976$, 0.982 and 0.983 , respectively (all p < 0.001, Figure 4D). Besides, compared with any single mRNA or clinical factors, the 7-mRNA-signature had better predictive value for relapse (all $\mathrm{p}<0.001$, Figure 4E-F). 
A

\section{TCGA cohort}

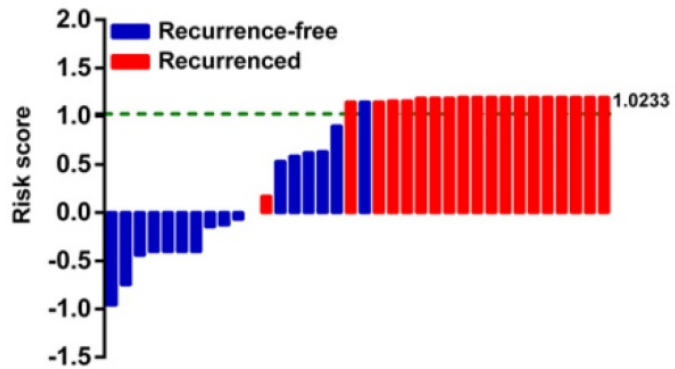

C

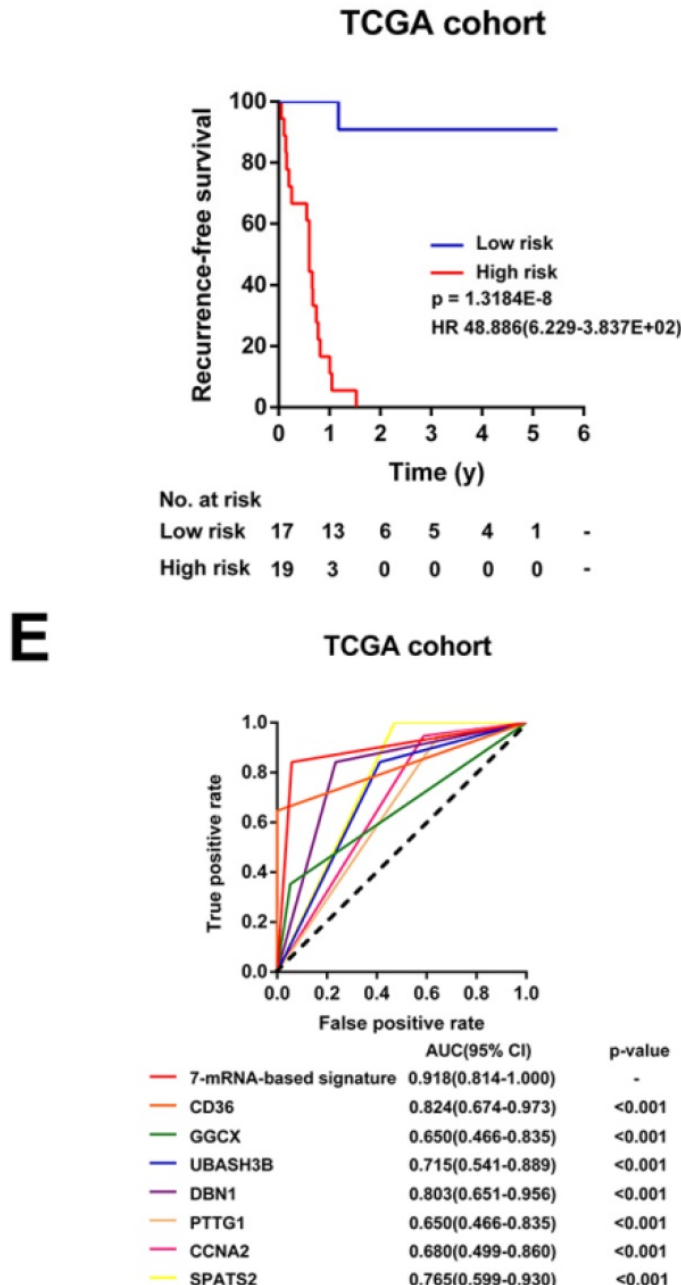

B

\section{TCGA cohort}

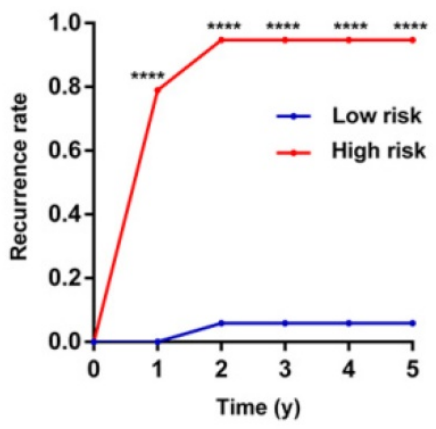

TCGA cohort

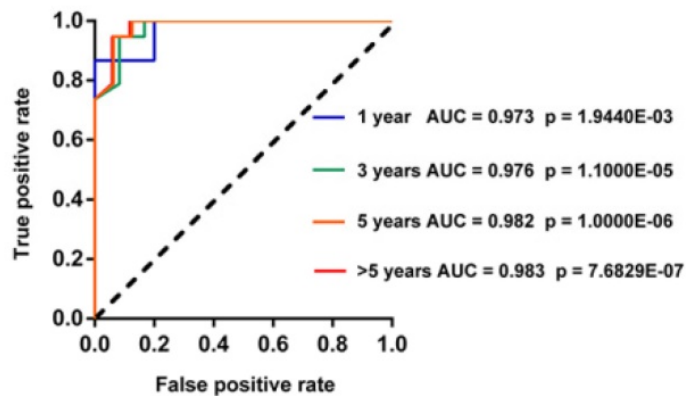

TCGA cohort

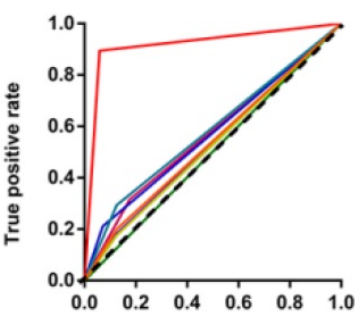

False positive rate

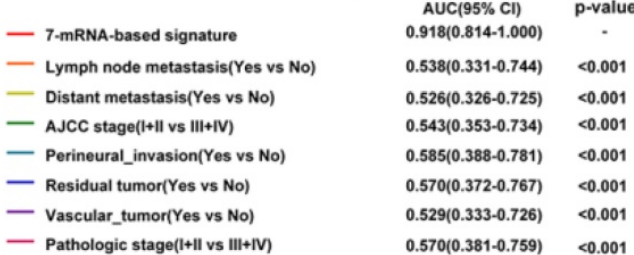

Figure 4. Evaluation of the risk score formula for relapse in the TCGA cohort. (A) Waterfall plots for distribution of risk score and relapse status of individual patients. (B) Recurrence rate between the high- and low-risk at the indicated time. (C) The Kaplan-Meier survival curve of recurrence-free for patients between two different groups. (D) Time-dependent ROC curve at 1 year, 3 years, 5 years and more than 5 years. (E) Comparison of prognostic accuracy between the signature and single mRNAs. (F) Comparison of prognostic accuracy between the signature and clinical characteristics. P-values were calculated using the log-rank test. HR, hazard ratio; AUC, area under ROC curve; RFS, recurrence-free survival. ****, $\mathrm{p}<0.001$.

In addition, univariable Cox analysis showed that only 7-mRNA signature were positively associated with CCA recurrence in TCGA cohort $(\mathrm{p}<$ 0.001, $\mathrm{HR}=48.886,95 \% \mathrm{CI}=6.229-383.657$, Table S3).
However, clinical association analyses showed that increased risk score was not related to clinical factors obviously, probably due to the small sample numbers (Table S4). 
Validation of the 7-mRNA signature for recurrence-free survival prediction in Ren Ji Cohort

To further verify whether this 7-mRNA classifier had a similar predictive ability in different CCA populations, we applied it to an independent cohort. From January 2012 to December 2017, forty-four CCA patients with complete clinicopathological information and prognostic outcomes were enrolled in our study at Ren Ji Hospital. We measured the expression levels of the $7 \mathrm{mRNAs}$ in $44 \mathrm{CCA}$ tumor samples by qRT-PCR assays (Figure S4). Then, the risk scores were calculated for every patient according to the expression status of these $7 \mathrm{mRNAs}$.

According to the optimal cutoff risk score determined by the ROC curve, patients were further divided into high- $(n=31)$ or low-risk $(n=13)$ groups. As shown in Figure 5A-B, patients with higher risk scores were more prone to recurrence after CCA resection. Survival analysis showed that patients in the high-risk group had obviously shorter RFS time than those in the low-risk group $(\mathrm{p}<0.001, \mathrm{HR}=$ 4.558, 95\% CI 1.829-11.357, Figure 5C). The AUCs of the time-dependent ROC curves between the 7-mRNA signature and RFS were 1.000 for 1 year, 0.958 for 3 years, 0.977 for 5 years and 0.979 for $>5$ years $(p=0.09$ at 1 year, others $p<0.01$, Figure $5 D)$. Moreover, the AUC of the 7-mRNA risk score model was significantly greater than any single mRNA or clinical factor (all $p<0.001$, Figure 5E-F).

Univariable Cox analyses of the Ren Ji cohort showed that CA19-9 levels, lymph node metastasis, and the 7-mRNA signature were significant factors that correlated with RFS of CCA (Table S5). Among these, the 7-mRNA signature was the most effective one to predict relapse of CCA in the Ren Ji cohort $(\mathrm{p}=$ $0.001, \mathrm{HR}=4.558,95 \%$ CI 1.829-11.357, Table S5). Furthermore, the multivariable Cox analysis showed that the 7-mRNA signature remained a powerful and independent factor for RFS after adjusting for other clinicopathological characteristics $(\mathrm{p}=0.008, \mathrm{HR}=$ $3.912,95 \% \mathrm{CI}=1.417-10.799$, Table S5). In addition, the 7-mRNA signature was found to be positively associated with the tumor size $(p=0.034)$ of CCA (Table S6).

\section{Stratification analysis of the 7-mRNA-based classifier in TCGA Cohort and Ren Ji Cohort}

To investigate the applicable CCA population of this 7-mRNA-based classifier, the 7-mRNA-signature based survival analyses were further performed in subgroups of patients with different clinical variables in the TCGA cohort and Ren Ji cohort (Figure 6-7, Figure S5-6).
For the TCGA cohort, upon stratified by individual clinicopathological features including gender, age, CA19-9 levels, tumor size, pathologic stage, and AJCC stage, the signature was still a clinically and statistically significant applicable model in predicting recurrence of CCA patients (Figure 6). However, because of the small sample size, for patients with positive of lymph node metastasis, distant metastasis, perineural invasion, residual tumor or vascular tumor, the 7-mRNA signature was a little powerless for relapse prediction (Figure S5).

Similarly, this 7-mRNA signature was a practical predictor that was independent of some clinicopathological characteristics like age, tumor thrombus and AJCC stage in the Renji cohort (Figure 7). For patients in subgroups of male, CA19-9 $\leq$ $37 \mathrm{ng} / \mathrm{ml}$, tumor size $\leq 5 \mathrm{~cm}$, mono-modular, negative of lymph node metastasis or distant metastasis, the 7-mRNA signature maintained its predictive value for recurrence-free survival (Figure S6B, S6C, S6E, S6G, S6I, and S6K). Unfortunately, the 7-mRNA signature lost the prognostic role for patients of female, CA19-9 $>37 \mathrm{ng} / \mathrm{ml}$, tumor size $>5 \mathrm{~cm}$, multi-modular, positive of lymph node metastasis or positive of distant metastasis, which might be due to the small sample number of these subgroups (Figure S6A, S6D, S6F, S6H, S6J and S6L).

\section{Discussion}

Cholangiocarcinoma is a fatal malignancy, which arising from varying locations within the biliary tree. Although surgical resection with curative intent is performed, the prognosis of patients with CCA remains poor owing to a high incidence of recurrence. Therefore, predicting recurrence is an arduous and urgent task [20].

At present, tumor biomarkers have been used to predict relapse in patients with CCA. Firstly, Carbohydrate antigen 19-9 (CA19-9) is a traditional serum biomarker used for CCA prognosis prediction. Some studies found that preoperative CA19-9 level higher than $100 \mathrm{U} / \mathrm{ml}$ were associated with a lower recurrence-free survival after operation. However, these tumor markers are not specific to CCA, and its elevation can be related to other diseases, such as bile duct obstruction or acute cholangitis [21]. Another study pointed out that preoperative serum CA19-9 level higher than $135 \mathrm{U} / \mathrm{ml}$ was a predictor for a lower survival rate [22]. Other serum markers, such as carcinoembryonic antigen (CEA), have proved to be overlapped with other diseases and showed low sensitivity and specificity. It has been reported in many studies that serum Cytokeratin 19 fragment 21-1 (CYFRA21-1) and CA-242 have higher specificities than CA19-9 for intrahepatic 
cholangiocarcinoma, but they haven't been used in clinical routine examination [23]. Some clinical features, such as tumor size and lymph node metastases, remain controversial in relapse prediction [24-26].
A

\section{Renji cohort}

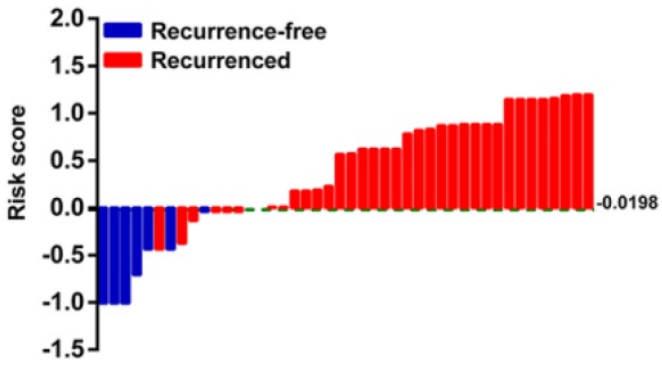

C

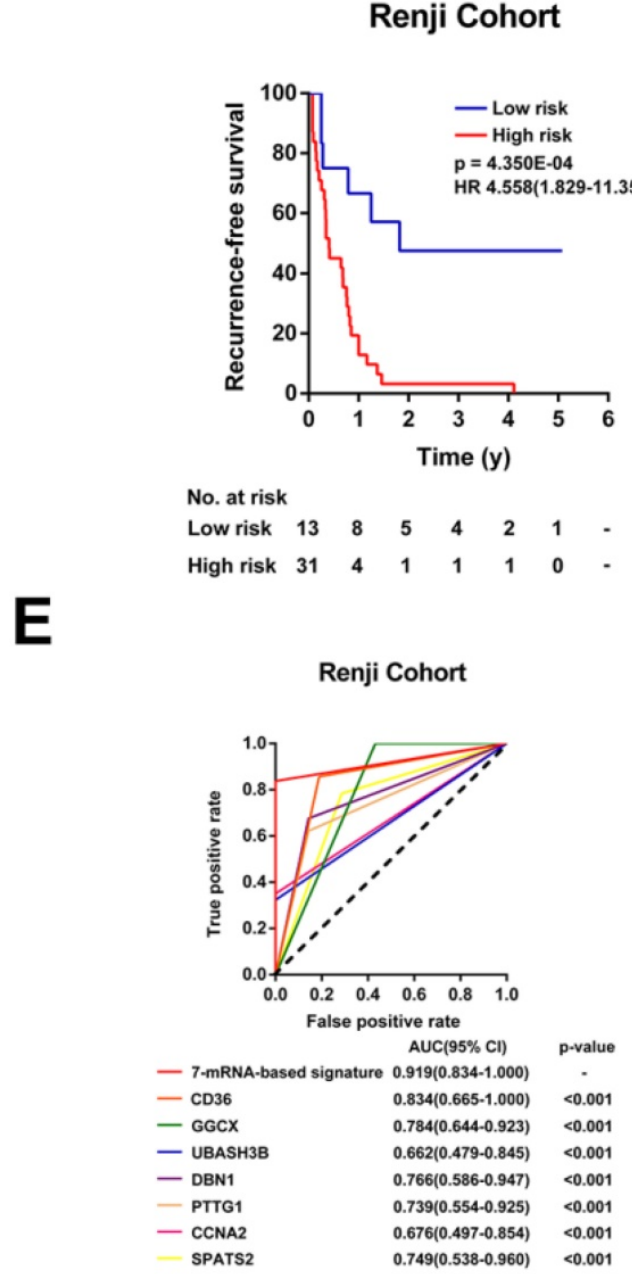

B

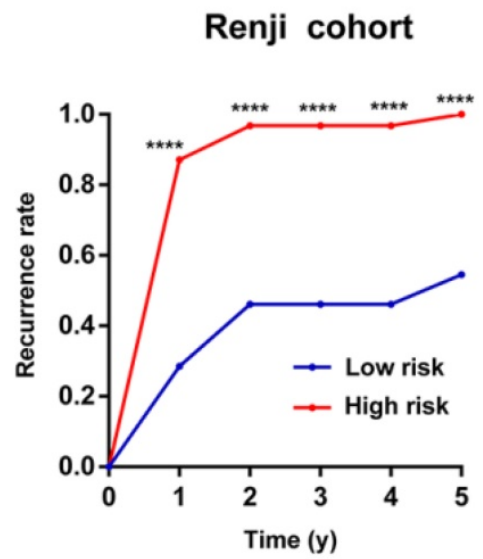

D

Renji cohort

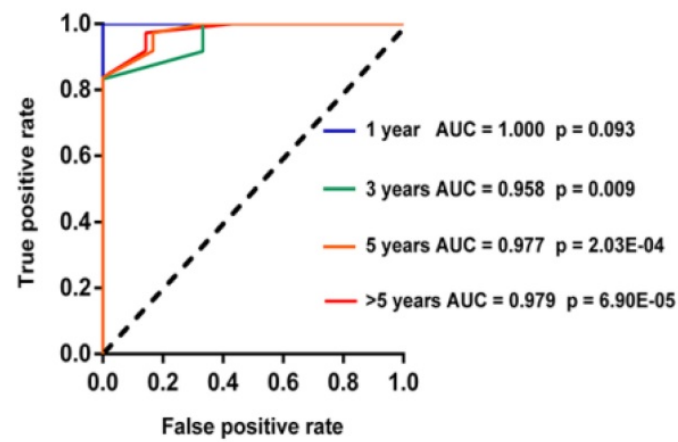

F

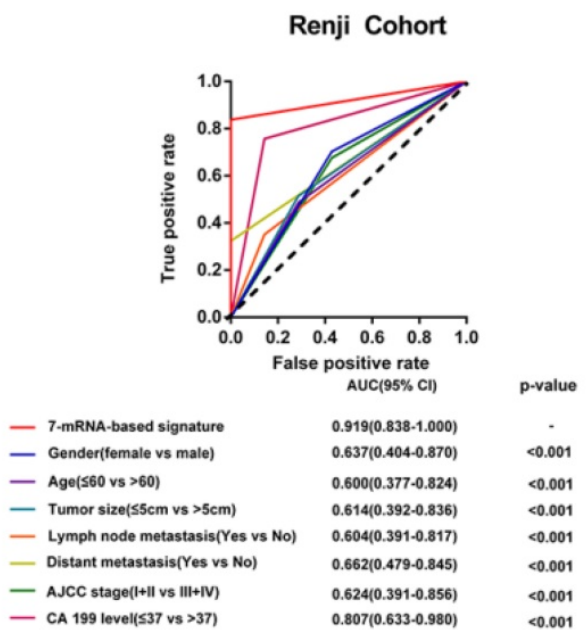

Figure 5. Validation of the 7-mRNA signature for relapse prediction in the Ren ji cohort. (A) Waterfall plots for distribution of risk score and relapse status of individual patients. (B) Recurrence rate between the high- and low-risk at the indicated time. (C) The Kaplan-Meier survival curve of recurrence-free for patients between two different groups. (D) Time-dependent ROC curve at 1 year, 3 years, 5 years and more than 5 years. (E) Comparison of prognostic accuracy between the signature and single mRNAs. $(F)$ Comparison of prognostic accuracy between the signature and clinical characteristics. P-values were calculated using the log-rank test. HR, hazard ratio; AUC, area under ROC curve; RFS, recurrence-free survival. ****, $p<0.001$. 


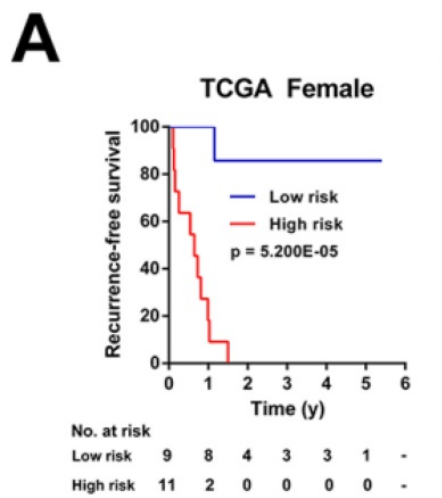

E TCGA CA199 $\leq 37$

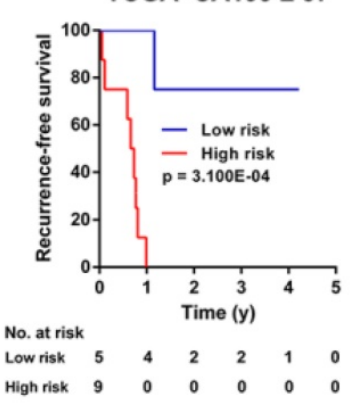

TCGA Pathologic I+II

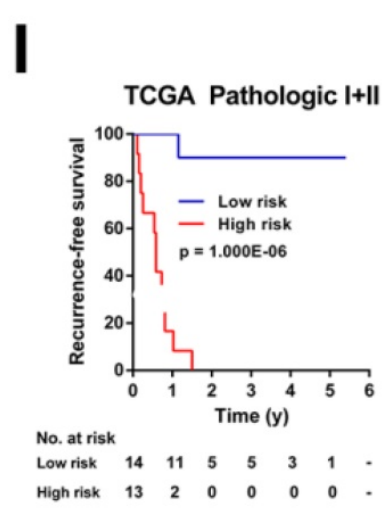

B

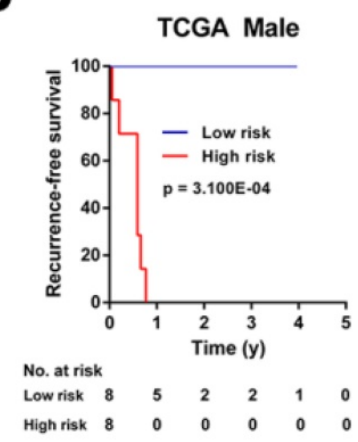

F

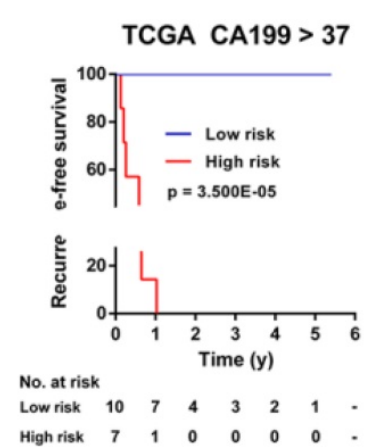

C

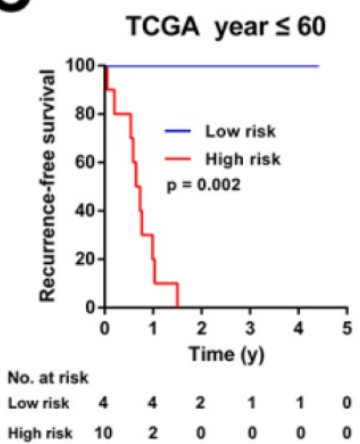

D

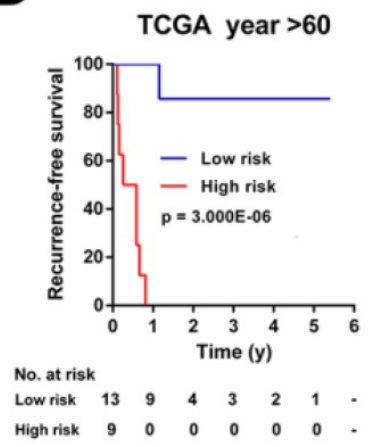

G

TCGA T1

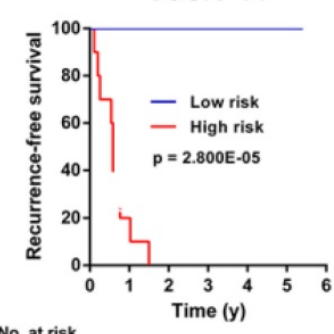

$\begin{array}{llllllll}\text { No. at risk } & & & & & \\ \text { Low risk } & 11 & 9 & 4 & 3 & 2 & 1\end{array}$.

$\begin{array}{llllllll}\text { High risk } & 10 & 1 & 0 & 0 & 0 & 0 & \text {. }\end{array}$

H

TCGA T2+T3+T4

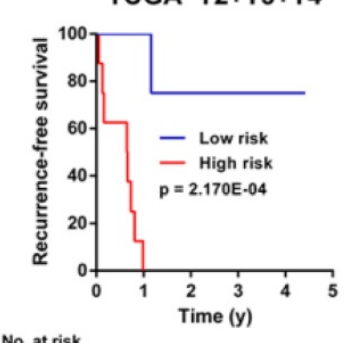

$\begin{array}{lllllll}\text { No. at risk } & & & & & \\ \text { Low risk } & 9 & 5 & 2 & 1 & 0 & 0\end{array}$

$\begin{array}{lllllll}\text { High risk } & 8 & 0 & 0 & 0 & 0 & 0\end{array}$

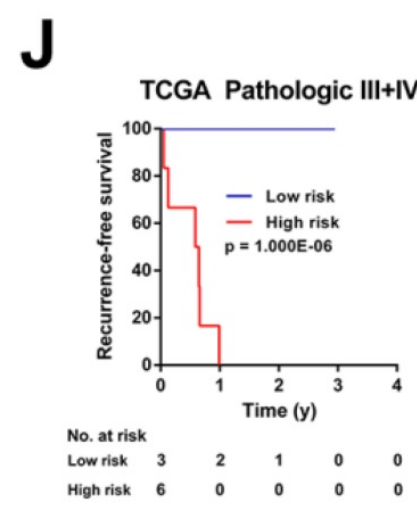

K

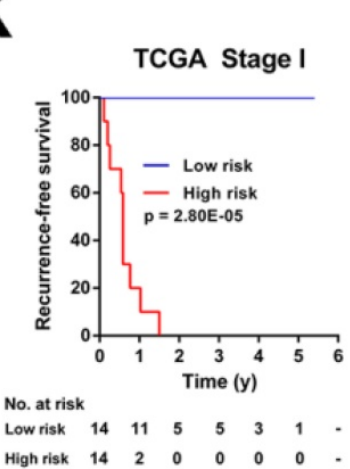

TCGA Stage $\|+|||+| V$

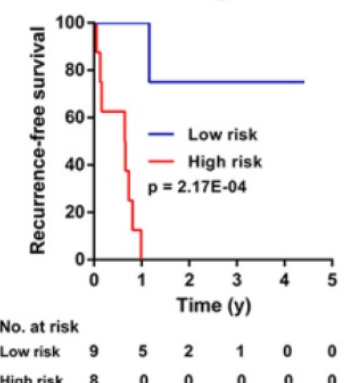

Figure 6. Kaplan-Meier survival analyses of the TCGA cohort, according to the 7-mRNA-based classifier stratified by clinicopathological characteristics. (A, B) Gender, (C, D) Age, (E, F) CA 199 levels, (G, H) Tumor size, (I, J) Pathologic stage, and (K, L) AJCC stage.

Currently, different versions of the AJCC or TNM staging system, along with the prognostic scoring systems have been widely used to evaluate the prognosis of CCA patients. However, these systems had some limitations in different subtypes of CCA [5], which might due to the ignorance of the different genetic and epigenetic backgrounds in tumor subtypes. In summary, postoperative prediction of CCA remains a problem, so, we conducted a multi-mRNA signature to accurately predict RFS for CCA patients.

In this study, we firstly selected datasets from GEO and TCGA. Then, we performed overlapping analysis with a strategic and stepwise method and finally obtain 194 DEGs in accordance with uniform standards $(\mathrm{p}<0.01$ and FC $>1.5$ or $<-1.5$ ). After screening, 127 DEGs with AUC $\geq 0.55$ were utilized to construct the LASSO COX regression model. Ten-fold cross-validation was used to select the best one with the minimum mean cross validation error from a series of models. Finally, we established a 7-mRNA-based signature risk score model for CCA patients. Cox univariable and multivariate analysis verified that 7-mRNA-signature was a powerful and independent prognostic factor for CCA patients. The time-dependent ROC curve demonstrated that this model was superior to other prognostic factors, such as CA19-9 levels and the AJCC staging system. 
A

Renji Year $\leq 60$

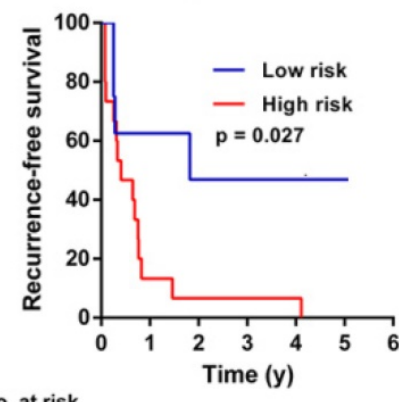

No. at risk

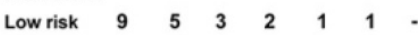

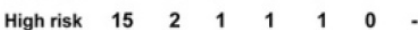

B

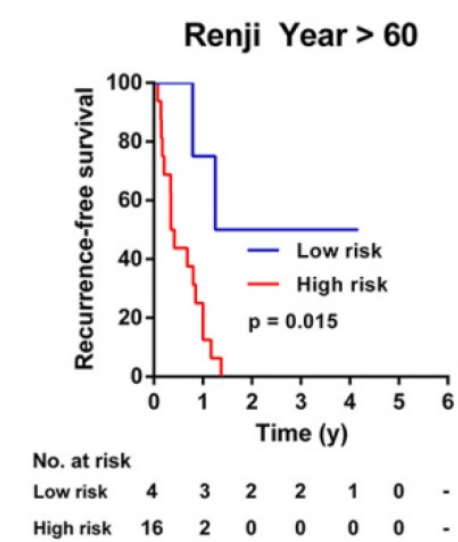

C

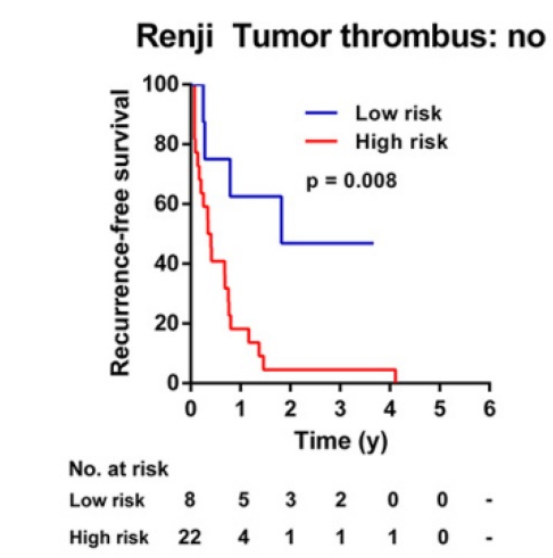

D
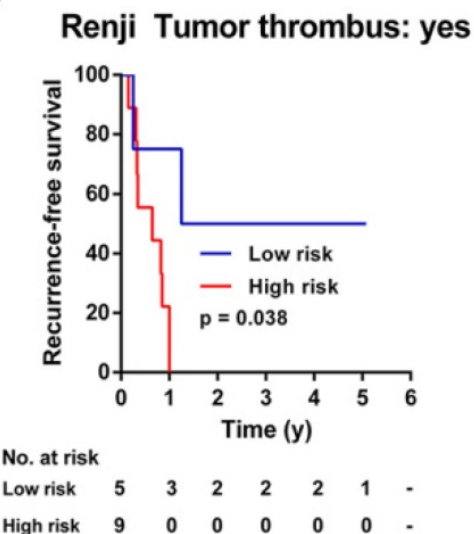

E

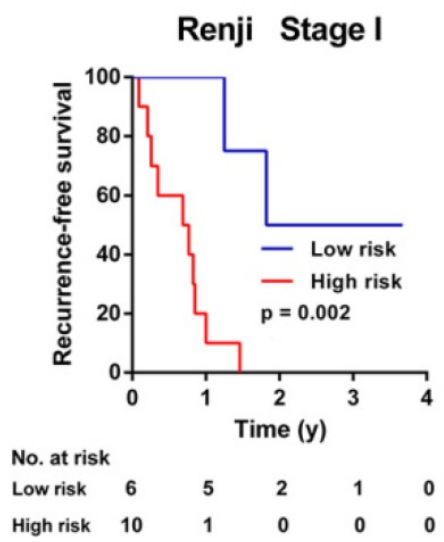

$\mathbf{F}$

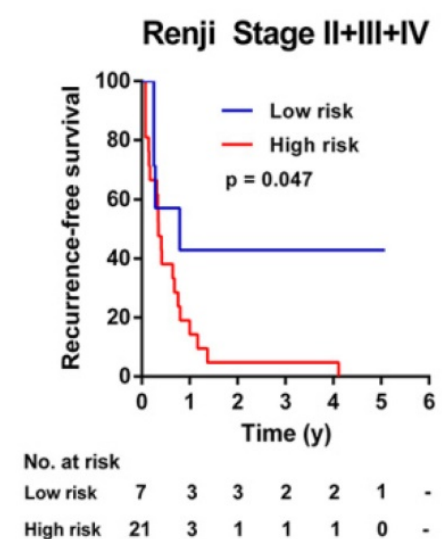

Figure 7. Kaplan-Meier survival analyses of the Ren Ji cohort, according to the 7-mRNA-based classifier stratified by clinicopathological characteristics. (A, B) Age, (C, D) Tumor thrombus, and (E, F) AJCC stage.

Meanwhile, we used two datasets to validate prognostic value of 7-mRNA-signature in relapse. The results proved that patients in the high-risk group tended to recurrence compared with the low-risk group.

Most genes included in the 7-mRNA signature have been experimentally researched in many studies. Uray et al. found that higher expression of CD36 could mediate TSP-1-stimulated apoptosis and promoted cellular adhesion in breast cancer cells, which might inhibit tumor migration and invasion in cholangiocarcinoma [27]. However, CD36 was a controversial indicator in pancreatic cancer, low expression of CD36 predicted lower TNM staging and CA19-9 levels, but larger tumor size and poor survival prognosis [28]. Ueda et al. reported that exon 2 deletion splice variant of GGCX could result in des-y-carboxy prothrombin (DCP) production in HCC cell lines [29], so, we guessed that GGCX metabolite DCP could play the same role in cholangiocarcinoma. Lee et al. found that phosphatase activity of UBASH3B could improve EGFR protein abundance, invasion, and metastasis in TNBC [30], and EGFR also played an important role in the progression of cholangiocarcinoma [1]. Iyama et al. reported that overexpression of DBN1 was associated with poor outcome of lung adenocarcinoma [31]. Meanwhile, DBN1 was reported to be involved in actin cytoskeleton reorganization, which had a vital role during cancer metastasis [32]. Ren et al. verified that pituitary tumor transforming gene-1 (PTTG1) was an independent prognostic factor and acted as an oncogene in colorectal cancer [33]. Furthermore, PTTG1 was known as a transcription factor, which exerts transcriptional activity either by directly binding to DNA or by interacting with proteins, including p53 [34], and p53 was consistently an important cancer suppressor gene in cholangiocarcinoma [35]. Li et al. found that cyclin A2 (CCNA2) promoted the EMT progression combined with MET/AKT/GSK-3b via the ROCK/AKT/ $\beta$-catenin pathway in bladder cancer [36], and aberrant activation of $\mathrm{Wnt} / \beta$-catenin signaling was observed in the majority of CCA [37]. In colorectal cancer, knockdown of CCNA2 inhibited cancer growth by impairing cell cycle [38]. Up-regulation of 
SPATS2 expression activated the STAT3 pathway and resulted in poor prognosis [39], and STAT3 had been implicated in carcinogenesis [40]. Besides, Takamochi et al. found that SPATS2 could help to differentiate squamous cell carcinoma from adenocarcinoma of the lung [41]. Unfortunately, the association of these 7 genes and cholangiocarcinoma recurrence has not been reported until now. However, CD36, GGCX, UBASH3B, DBN1, PTTG1, CCNA2, and SPATS2 are found in other tumors to regulate tumor progression, which may also regulate cholangiocarcinoma progression and affect the recurrence of CCA. Therefore, the potential mechanisms of these 7 genes with CCA recurrence need to be studied furthermore.

However, there were still some limitations to our study. Firstly, we used a relatively small sample size of CCAs. Secondly, besides mRNA, the predictive value of microRNA, IncRNA, and CpG in tumor prognosis had been validated. Multi-dimensional data analysis integrated with mRNA, microRNA, lncRNA, CpG might further increase the predictive efficiency [42-44]. Finally, cell functions and molecule mechanisms for the $7 \mathrm{mRNAs}$ had not been explored in our study.

\section{Abbreviations}

mRNA: messenger RNA; CCA: Cholangiocarcinoma; GEO: Gene Expression Omnibus; TCGA: The Cancer Genome Atlas; LASSO: Least absolute shrinkage and selection operator; HR: hazard ratio; CI: confidence interval; AJCC: American Joint Committee on Cancer; KRAS: KRAS proto-oncogene GTPas; EGFR: epidermal growth factor receptor; ERBB2: erb-b2 receptor tyrosine kinase 2; ROC: receiver operating characteristic; RFS: recurrence-free survival; GO: gene ontology; KEGG: Kyoto Encyclopedia of Genes and Genomes; DEGs: differentially expressed genes; FC: fold change; BP: biological processes; CC: cellular component; MF: molecular function; AUC: Area Under Curve; PCR: Polymerase Chain Reaction; CD36: CD36 molecule; GGCX: Gamma-glutamyl carboxylase; UBASH3B: ubiquitin associated and $\mathrm{SH} 3$ domain containing $\mathrm{B}$; DBN1: drebrin 1; PTTG1: pituitary tumor transforming gene-1; CCNA2: cyclin A2; SPATS2: spermatogenesis associated serine rich 2; CA19-9: Carbohydrate antigen 19-9; PSC: primary sclerosing cholangitis; CEA: carcinoembryonic antigen; CYFRA21-1: Cytokeratin 19 fragment 21-1.

\section{Supplementary Material}

Supplementary figures and tables. http://www.ijbs.com/v16p0869s1.pdf

\section{Acknowledgments}

This work was supported by the National Key Sci-Tech Special Project of China (2018ZX10723204006-004) to X Kong. The National Natural Science Foundation of China (81873582 and 81670562 to $X$ Kong, 81670598 to Q Xia, 31870905 and 31671453 to H. $\mathrm{Wu})$.

\section{Author contributions}

GH had the idea and drafted the manuscript. CJ, WX and WBR analyzed the data and prepared the figures and tables. KXM, GYQ and WHL provided clinical information and statistical advice. QXY, LX and WF prepared clinical samples, extracted RNA and performed qRT-PCR assays. SXH, XQ and KXN supervised the study and revised the manuscript. All authors approved the final version of the manuscript.

\section{Competing Interests}

The authors have declared that no competing interest exists.

\section{References}

1. Razumilava N: Gores GJ. Cholangiocarcinoma. Lancet. 2014; 383: 2168-79.

2. Bridgewater J: Galle PR: Khan SA: Llovet JM: Park JW: Patel T: et al. Guidelines for the diagnosis and management of intrahepatic cholangiocarcinoma. J Hepatol. 2014; 60: 1268-89.

3. Khan SA: Davidson BR: Goldin RD: Heaton N: Karani J: Pereira SP: et al. Guidelines for the diagnosis and treatment of cholangiocarcinoma: an update. Gut. 2012; 61: 1657-69.

4. Khan SA: Emadossadaty S: Ladep NG: Thomas HC: Elliott P: Taylor-Robinson SD: et al. Rising trends in cholangiocarcinoma: is the ICD classification system misleading us? J Hepatol. 2012; 56: 848-54

5. Blechacz B: Komuta M: Roskams T: Gores GJ. Clinical diagnosis and staging of cholangiocarcinoma. Nat Rev Gastroenterol Hepatol. 2011; 8: 512-22.

6. Sirica AE. Cholangiocarcinoma: molecular targeting strategies for chemoprevention and therapy. Hepatology. 2005; 41: 5-15.

7. Zabron A: Edwards RJ: Khan SA. The challenge of cholangiocarcinoma: dissecting the molecular mechanisms of an insidious cancer. Dis Model Mech. 2013; 6: 281-92.

8. Sia D: Hoshida Y: Villanueva A: Roayaie S: Ferrer J: Tabak B: et al. Integrative molecular analysis of intrahepatic cholangiocarcinoma reveals 2 classes that have different outcomes. Gastroenterology. 2013; 144: 829-40.

9. Andersen JB: Spee B: Blechacz BR: Avital I: Komuta M: Barbour A: et al. Genomic and genetic characterization of cholangiocarcinoma identifies therapeutic targets for tyrosine kinase inhibitors. Gastroenterology. 2012; 142: 1021-31.e15.

10. Edgar R: Domrachev M: Lash AE. Gene Expression Omnibus: NCBI gene expression and hybridization array data repository. Nucleic Acids Res. 2002; 30: $207-10$

11. Deng M: Bragelmann J: Schultze JL: Perner S. Web-TCGA: an online platform for integrated analysis of molecular cancer data sets. BMC Bioinformatics. 2016; 17: 72

12. Barrett T: Wilhite SE: Ledoux P: Evangelista C: Kim IF: Tomashevsky M: et al. NCBI GEO: archive for functional genomics data sets--update. Nucleic Acids Res. 2013; 41: D991-5.

13. Shi G: Zhang J: Lu Z: Liu D: Wu Y: Wu P: et al. A novel messenger RNA signature as a prognostic biomarker for predicting relapse in pancreatic ductal adenocarcinoma. Oncotarget. 2017; 8: 110849-60.

14. Zhu X: Tian X: Yu C: Shen C: Yan T: Hong J: et al. A long non-coding RNA signature to improve prognosis prediction of gastric cancer. Mol Cancer. 2016; $15: 60$.

15. Tibshirani R. The lasso method for variable selection in the Cox model. Stat Med. 1997; 16: 385-95.

16. Gui J: Li H. Penalized Cox regression analysis in the high-dimensional and low-sample size settings: with applications to microarray gene expression data. Bioinformatics. 2005; 21: 3001-8

17. Meng ZW: Pan W: Hong HJ: Chen JZ: Chen YL. Macroscopic types of intrahepatic cholangiocarcinoma and the eighth edition of AJCC/UICC TNM staging system. Oncotarget. 2017; 8: 101165-74.

18. Hoo ZH: Candlish J: Teare D. What is an ROC curve? Emerg Med J. 2017; 34: $357-9$. 
19. Kamarudin AN: Cox T: Kolamunnage-Dona R. Time-dependent ROC curve analysis in medical research: current methods and applications. BMC Med Res Methodol. 2017; 17: 53.

20. Zhang XF: Beal EW: Bagante F: Chakedis J: Weiss M: Popescu I: et al. Early versus late recurrence of intrahepatic cholangiocarcinoma after resection with curative intent. Br J Surg. 2018; 105: 848-56.

21. Tamandl D: Herberger B: Gruenberger B: Puhalla H: Klinger M: Gruenberger $\mathrm{T}$. Influence of hepatic resection margin on recurrence and survival in intrahepatic cholangiocarcinoma. Ann Surg Oncol. 2008; 15: 2787-94.

22. Uchiyama K: Yamamoto M: Yamaue H: Ariizumi S: Aoki T: Kokudo N: et al. Impact of nodal involvement on surgical outcomes of intrahepatic cholangiocarcinoma: a multicenter analysis by the Study Group for Hepatic Surgery of the Japanese Society of Hepato-Biliary-Pancreatic Surgery. J Hepatobiliary Pancreat Sci. 2011; 18: 443-52

23. Uenishi T: Yamazaki O: Tanaka H: Takemura S: Yamamoto T: Tanaka S: et al. Serum cytokeratin 19 fragment (CYFRA21-1) as a prognostic factor in intrahepatic cholangiocarcinoma. Ann Surg Oncol. 2008; 15: 583-9.

24. Choi SB: Kim KS: Choi JY: Park SW: Choi JS: Lee WJ: et al. The prognosis and survival outcome of intrahepatic cholangiocarcinoma following surgical resection: association of lymph node metastasis and lymph node dissection with survival. Ann Surg Oncol. 2009; 16: 3048-56.

25. Tamandl D: Kaczirek K: Gruenberger B: Koelblinger C: Maresch J: Jakesz R: et al. Lymph node ratio after curative surgery for intrahepatic cholangiocarcinoma. Br J Surg. 2009; 96: 919-25.

26. Endo I: Gonen M: Yopp AC: Dalal KM: Zhou Q: Klimstra D: et al. Intrahepatic cholangiocarcinoma: rising frequency: improved survival: and determinants of outcome after resection. Ann Surg. 2008; 248: 84-96.

27. Uray IP: Liang Y: Hyder SM. Estradiol down-regulates CD36 expression in human breast cancer cells. Cancer Lett. 2004; 207: 101-7.

28. Jia S: Zhou L: Shen T: Zhou S: Ding G: Cao L. Down-expression of CD36 in pancreatic adenocarcinoma and its correlation with clinicopathological features and prognosis. J Cancer. 2018; 9: 578-83.

29. Ueda N: Shiraha H: Fujikawa T: Takaoka N: Nakanishi Y: Suzuki M: et al. Exon 2 deletion splice variant of gamma-glutamyl carboxylase causes des-gamma-carboxy prothrombin production in hepatocellular carcinoma cell lines. Mol Oncol. 2008; 2: 241-9.

30. Lee ST: Feng M: Wei Y: Li Z: Qiao Y: Guan P: et al. Protein tyrosine phosphatase UBASH3B is overexpressed in triple-negative breast cancer and promotes invasion and metastasis. Proc Natl Acad Sci U S A. 2013; 110: 11121-6.

31. Iyama S: Ono M: Kawai-Nakahara H: Husni RE: Dai T: Shiozawa T: et al. Drebrin: A new oncofetal biomarker associated with prognosis of lung adenocarcinoma. Lung Cancer. 2016; 102: 74-81.

32. Lin Q: Tan HT: Lim TK: Khoo A: Lim KH: Chung MC. iTRAQ analysis of colorectal cancer cell lines suggests Drebrin (DBN1) is overexpressed during liver metastasis. Proteomics. 2014; 14: 1434-43.

33. Ren Q: Jin B. The clinical value and biological function of PTTG1 in colorectal cancer. Biomed Pharmacother. 2017; 89: 108-15.

34. Zhang Z: Jin B: Jin Y: Huang S: Niu X: Mao Z: et al. PTTG1: A novel androgen responsive gene is required for androgen-induced prostate cancer cell growth and invasion. Exp Cell Res. 2017; 350: 1-8.

35. Khan SA: Thomas HC: Toledano MB: Cox IJ: Taylor-Robinson SD. p53 Mutations in human cholangiocarcinoma: a review. Liver Int. 2005; 25: 704-16.

36. Li J: Ying Y: Xie H: Jin K: Yan H: Wang S: et al. Dual regulatory role of CCNA2 in modulating CDK6 and MET-mediated cell-cycle pathway and EMT progression is blocked by miR-381-3p in bladder cancer. Faseb j. 2019; 33: 1374-88

37. Wang W: Smits R: Hao H: He C. Wnt/beta-Catenin Signaling in Liver Cancers. Cancers (Basel). 2019; 11

38. Gan Y: Li Y: Li T: Shu G: Yin G. CCNA2 acts as a novel biomarker in regulating the growth and apoptosis of colorectal cancer. Cancer Manag Res. 2018; 10: 5113-24.

39. Damas ND: Marcatti M: Come C: Christensen LL: Nielsen MM: Baumgartner R: et al. SNHG5 promotes colorectal cancer cell survival by counteracting STAU1-mediated mRNA destabilization. Nat Commun. 2016; 7: 13875.

40. Sansone P: Bromberg J. Targeting the interleukin-6/Jak/stat pathway in human malignancies. J Clin Oncol. 2012; 30: 1005-14.

41. Takamochi K: Ohmiya H: Itoh M: Mogushi K: Saito T: Hara K: et al. Novel biomarkers that assist in accurate discrimination of squamous cell carcinoma from adenocarcinoma of the lung. BMC Cancer. 2016; 16: 760.

42. Li Z: Shen J: Chan MT: Wu WK. The role of microRNAs in intrahepatic cholangiocarcinoma. J Cell Mol Med. 2017; 21: 177-84

43. Dai K: Quan J: Yan F: Jin X: Pan X: Song X: et al. IncRNAs as potential molecular biomarkers in the clinicopathology and prognosis of cholangiocarcinoma: a systematic review and meta-analysis. Onco Targets Ther. 2019; 12: 1905-15.

44. Lee S: Kim WH: Jung HY: Yang MH: Kang GH. Aberrant CpG island methylation of multiple genes in intrahepatic cholangiocarcinoma. Am J Pathol. 2002; 161: 1015-22. 